\title{
Fallow time determination in dentistry using aerosol measurement in mechanically and non-mechanically ventilated environments
}

\author{
Shakeel Shahdad ${ }_{1}{ }^{* 1}$ Annika Hindocha, ${ }^{2}$ Tulsi Patel, ${ }^{2}$ Neil Cagney, ${ }_{1}$ Jens-Dominik Mueller, ${ }^{4}$ Amine Koched ${ }_{1}^{5}$ Noha \\ Seoudi, ${ }^{6}$ Claire Morgan, ${ }^{7}$ Padhraig S. Fleming ${ }^{8}$ and Ahmed Riaz Din ${ }^{9}$
}

\section{Key points}

Mechanical ventilation for aerosol generating procedures (AGPs) should be gold standard; where not available or practical, then the use of natural ventilation with extraoral suction helps reduce fallow time.
AGPs can be carried out in open bay environments with a minimum of six air changes per hour of mechanical ventilation.
Four-handed dentistry with high-volume suction and a saliva ejector are essential mitigating factors during AGPs.

\begin{abstract}
Aim To calculate fallow time (FT) required following dental aerosol generating procedures (AGPs) in both a dental hospital (mechanically ventilated) and primary care (non-mechanically ventilated). Secondary outcomes were to identify spread and persistence of aerosol in open clinics compared to closed surgeries (mechanically ventilated environment), and identify if extraoral scavenging (EOS) reduces FT and production of aerosol.
\end{abstract}

Methods In vitro simulation of fast handpiece cavity preparations using a manikin was conducted in a mechanically and non-mechanically ventilated environment using Optical Particle Sizer and NanoScan at baseline, during the procedure and fallow period.

Results AGPs carried out in the non-mechanically, non-ventilated environment failed to achieve baseline particle levels after one hour. In contrast, when windows were opened after AGPs, there was an immediate reduction in all particle sizes. In mechanically ventilated environments, the baseline levels of particles were very low and particle count returned to baseline within ten minutes following the AGP. There was no detectable difference between particles in mechanically ventilated open bays and closed surgeries. The effect of the EOS on reducing the particle count was greater in the non-mechanically ventilated environment; additionally, it also reduced the spikes in particle counts in mechanically ventilated environments.

Conclusion High-efficiency particulate, air-filtered mechanical ventilation, along with mitigation (high-volume suction), resulted in reduction of fallow time (ten minutes). Non-ventilated rooms failed to reach baseline level even after one hour of fallow time. There was no difference in particle counts in open bays or closed surgeries in mechanically ventilated settings with an extraoral suction device reducing particulate spikes. This study confirms that AGPs are not recommended in dental surgeries where no ventilation is possible.

'Honorary Clinical Professor in Oral Rehabilitation \& Implantology and Consultant in Restorative Dentistry, Barts and The London School of Medicine and Dentistry, Queen Mary University of London, Barts Health NHS Trust, The Royal London Dental Hospital, London, UK; ${ }^{2}$ Dental Core Trainee, Restorative Dentistry and General Duties, Barts Health NHS Trust, The Royal London Dental Hospital, London, UK; ${ }^{3}$ Lecturer, School of Engineering and Materials Science, Faculty of Science and Engineering, Queen Mary University of London, Mile End Road, London, E1 4NS, UK; ${ }^{4 R e a d e r ~ i n ~ C o m p u t a t i o n a l ~ F l u i d ~ D y n a m i c s ~}$ and Optimisation, School of Engineering and Materials Science, Queen Mary University of London, Mile End Road, London, E1 4NS, UK; ${ }^{5}$ Research and Analytical Application Specialist, TSI Inc, USA; ${ }^{6}$ Senior Clinical Lecturer in Oral Microbiology, Centre for Oral Immunobiology and Regenerative Medicine, Barts and the London School of Medicine and Dentistry, Queen Mary University of London, London, E1 2AD, UK; ${ }^{7}$ Consultant in Restorative Dentistry, Barts Health NHS Trust, The Royal London Dental Hospital, London, UK; ${ }^{8 P r o f e s s o r ~ i n ~ O r t h o d o n t i c s ~ a n d ~ C o n s u l t a n t ~ i n ~ O r t h o d o n t i c s, ~ B a r t s ~ a n d ~ T h e ~ L o n d o n ~ S c h o o l ~}$ of Medicine and Dentistry, Queen Mary University of London, Barts Health NHS Trust, The Royal London Dental Hospital, London, UK; ${ }^{9}$ Post-CCST Speciality Registrar in Orthodontics, Barts Health NHS Trust, The Royal London Dental Hospital, London, UK.

${ }^{*}$ Correspondence to: Shakeel Shahdad

Email address: shakeel.shahdad@nhs.net

Refereed Paper.

Accepted 19 July 2021

https://doi.org/10.1038/s41415-021-3369-1

\section{Introduction}

COVID-19 has the potential to spread during dental procedures through a number of routes. Attention has focused on the spread via droplets or 'splatter' that can either impact directly on the face of a susceptible person or be deposited on a surface. ${ }^{1,2}$ However, there is increasing evidence that aerosols, particularly when highly concentrated in enclosed environments, may play an important role in disease transmission., ${ }^{3,45}$ Aerosols in dental procedures are typically defined as particles smaller than $5 \mu \mathrm{m}$ that can remain suspended in air for hours. ${ }^{3}$ 
There have been extensive investigations attempting to characterise the potential for infection from aerosol to occur in dentistry. ${ }^{6,7,8}$ Many researchers have focused on measuring bioaerosol using cultures to quantify the amounts of bacteria or fungi deposited on surfaces. However, this approach relies on bioaerosols settling onto the surface and cannot account for the particles that remain suspended in the air or those removed through ventilation. ${ }^{9,10,11,12,13,14,15}$

Other researchers have added dye or fluorescent marker to the water lines to examine the distribution of splatter and detect deposits as small as $1,000 \mu \mathrm{m}^{2}$ in area, although this dimension exceeds what is typically classed as an aerosol. ${ }^{1,2,16,17}$ Small particles $(<16-27 \mu \mathrm{m})$ deposited on microscope slides have also been studied; however, this did not account for aerosols that did not settle during the experiment. ${ }^{18}$

In the past decade, there have been a small number of studies using particle counters to directly sample the concentration of aerosols suspended in the air, ${ }^{19}$ although the focus has often been on the nanoparticles released from dental composite materials rather than on the potential spread of infection. ${ }^{20}$

As a result of risks associated with COVID19 , routine use of fallow time (FT) to allow for settling of suspended aerosol has been recommended following aerosol generating procedures (AGPs). Routine adoption of FTs may limit the capacity for the provision of dental care. However, there has been little consistency in the definition of an AGP or indeed on the necessity for and duration of FT following AGPs. ${ }^{21,22}$ Due to the lack of experimental data on aerosols produced during dental procedures, there is no consensus on the FT required after AGPs. Previous guidance from Public Health England, based on the New and Emerging Respiratory Virus Threats Advisory Group, recommended a FT of 60 minutes in a single room with six air changes per hour $(\mathrm{ACH})$ following AGPs. ${ }^{23,24} \mathrm{~A}$ recent rapid review of international dental guidance documents found that most did not refer to a FT. When FT was recommended, this varied between 2-180 minutes. ${ }^{25}$ The median FT was 15 minutes for 'non-COVID-19' patients and 20 minutes for confirmed or suspected COVID19 patients. $^{25}$

There has been an increase in availability of extraoral scavenger (suction) devices (EOSs) on the market since the beginning of the
COVID-19 pandemic. In a recently published study, EOSs reduced the mean intensity of contamination and frequency of splatter during most of the simulated procedures in both open clinic and closed surgery in a dental hospital, with mechanical ventilation at six ACH. ${ }^{2,21}$ However, the effect of EOSs on aerosol after AGPs is yet to be proven. Therefore, the primary aim of this study was to calculate the FT required for aerosols produced during various simulated AGPs to return to baseline levels in a dental hospital (mechanical ventilation) and a primary care setting (non-mechanical ventilation).

Secondary aims were to: i) identify if an EOS reduces the production of aerosol and FT required following AGPs in dentistry; and ii) identify if spread and persistence of aerosol generated in an open clinic was worse than a closed surgery in a mechanically ventilated environment.

\section{Methodology}

AGPs were simulated on a dental manikin in a multi-chair open clinic and closed surgery in a dental teaching hospital (The Royal London Dental Hospital, London, UK). During all procedures, mechanical ventilation with six $\mathrm{ACH}$ involving a centralised air exchange system remained operational. The procedures were repeated in a private dental clinic (Specialist Dental Services, 94 Harley Street, London, UK) with and without natural (nonmechanical) ventilation.

AGPs were carried out using a protocol using an air turbine (W\&H Synea Turbine TA-98LED, Bürmoos, Austria) for 20 minutes while simulating cavity preparation of tooth 36 , and crown preparation of 31 and 21 on thermoplastic teeth. Handpieces were operated at approximately 360,000 RPM with air and water coolant at maximum flow.

All procedures were carried out using a four-handed dentistry technique which included the assistant operating high-volume suction (HVS) and a saliva ejector (SE). The procedures were repeated three times by the same operators to reduce performance bias.

The procedure was repeated with an EOS which was placed at the 5 o'clock position, $15 \mathrm{~cm}$ from the mouth of the manikin and operated at maximum flow rate (TM10, TopMed Dental Lighting Co. Ltd, Foshan, China; specified flow rate $310 \mathrm{~m}^{3} / \mathrm{h}$ ).

Aerosol measurement with particle assessment was undertaken using both an optical particle scanner (Optical Particle Sizer 3330, TSI Inc., Minnesota, USA), which measured particles in the range of $0.3-10$ $\mu \mathrm{m}$, and a spectrometer particle scanner (NanoScan SMPS Nanoparticle Sizer 3910, TSI Inc., Minnesota, USA), which spanned the range $10 \mathrm{~nm}-0.365 \mu \mathrm{m}$ and operated as described previously. ${ }^{21}$ Briefly, the sampling inlets were placed adjacent to the manikin's mouth in the 7 oclock position $(8 \mathrm{~cm}$ from tooth 21) for all procedures (Fig. 1). Using both counters, it was possible to measure particles in 26 bins, ranging from $10 \mathrm{~nm}$ to $10 \mu \mathrm{m}$ in diameter. As a single SARS$\mathrm{CoV}-2$ virion is approximately $80-100 \mathrm{~nm}$ in diameter, formation of particles smaller than $80 \mathrm{~nm}$ was deemed to be irrelevant to virus transmission and was discarded. In order to reduce the remaining dataset, the particle counts were combined into four categories: 'very small' (0.08-0.26 $\mu \mathrm{m})$, 'small' (0.27-0.90 $\mu \mathrm{m})$, 'medium' (0.91-2.70 $\mu \mathrm{m})$ and 'large' (2.71-10 $\mu \mathrm{m})$.

Measurements were recorded continuously and each sample cycle took one minute to complete (Aerosol Instrument Manager 10.3.1.0 and NanoScan Manager 1.0.0.19, TSI Inc., Minnesota, USA). All experiments

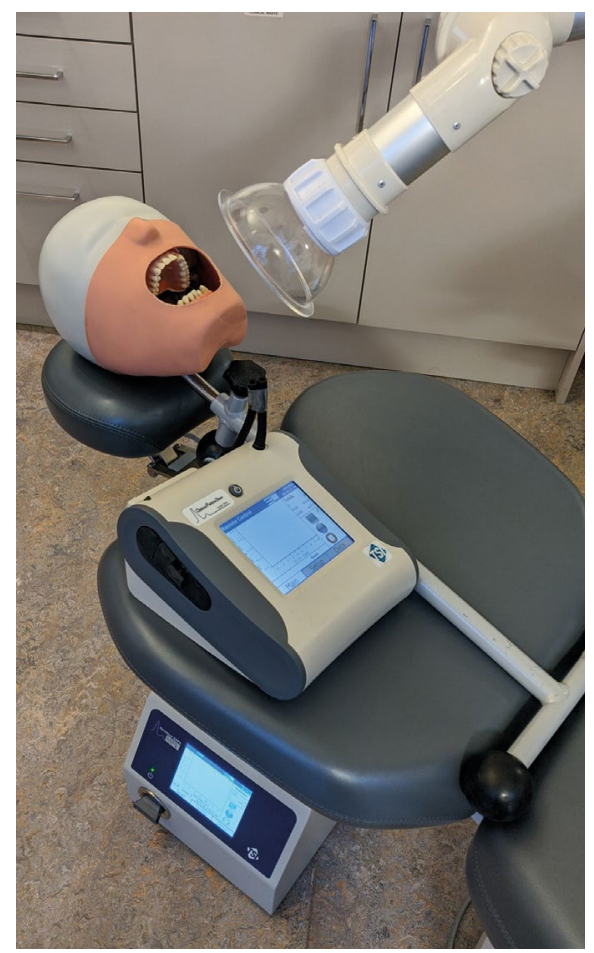

Fig. 1 Optical Particle Sizer 3330 (TSI Inc Minnesota, USA) (on dental chair) and NanoScan SMPS Nanoparticle Sizer 3910 (TSI Inc Minnesota, USA) (on floor). The sampling inlets placed adjacent to the manikin's mouth in the $7 o^{\prime}$ clock position $(8 \mathrm{~cm}$ from tooth 21$)$ 
included a pre-operative measurement of ten minutes to allow for a baseline atmosphere or characteristics recording, followed by the procedure and finally by a post-procedure FT. The post-procedure FT was initially chosen as 60 minutes. However, pilot results indicated that this could be reduced to 30 minutes for procedures carried out in the mechanically ventilated setting.

For procedures involving the EOS, the preprocedure particle measurement was recorded with ten minutes of no activity followed by ten minutes with the EOS functioning alone to evaluate the effect of the EOS on baseline measurements. This was then followed by the AGP and the post-procedure FT as above.

Only the operator and assistant were present in the room before the procedure
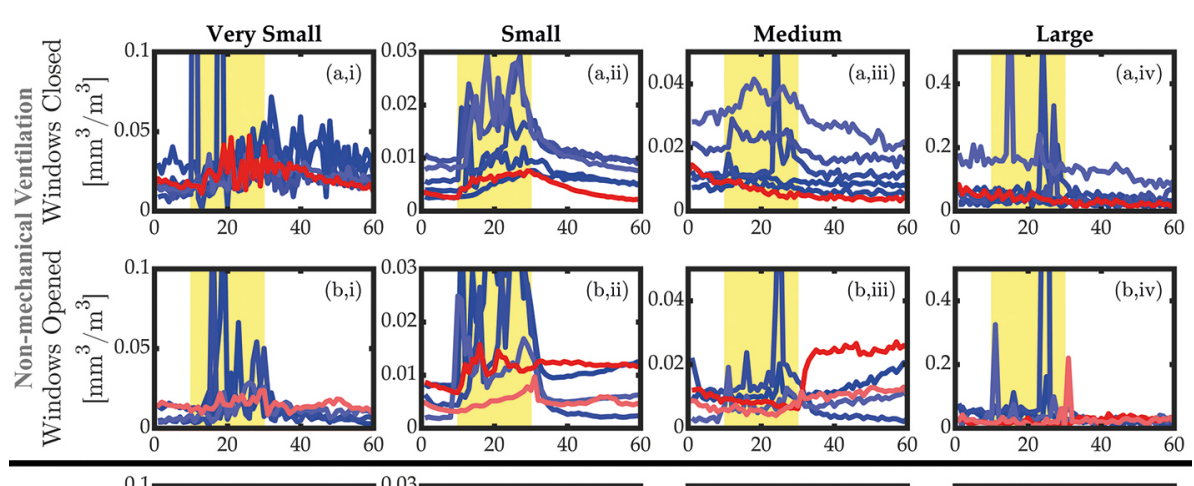

and they left immediately after completion of the procedure. The door was kept shut in the closed surgery during all times. In EOS procedures, the device was left functioning during the post-procedure FT. The operator and assistant wore fluid-resistant surgical masks during the procedures and talking was allowed pre-procedure to simulate a typical dental appointment.

In order to study the spread of aerosol, open clinic experiments were repeated with the particle-measuring equipment positioned at varying distances from the manikin, including an adjacent bay (at a distance of 1.7 $\mathrm{m}$ and over a partition wall measuring $1.2 \mathrm{~m}$ tall) and opposite bays (at a distance of $1.7 \mathrm{~m}$ with no intersecting partition).

In order to identify the aerosols actually
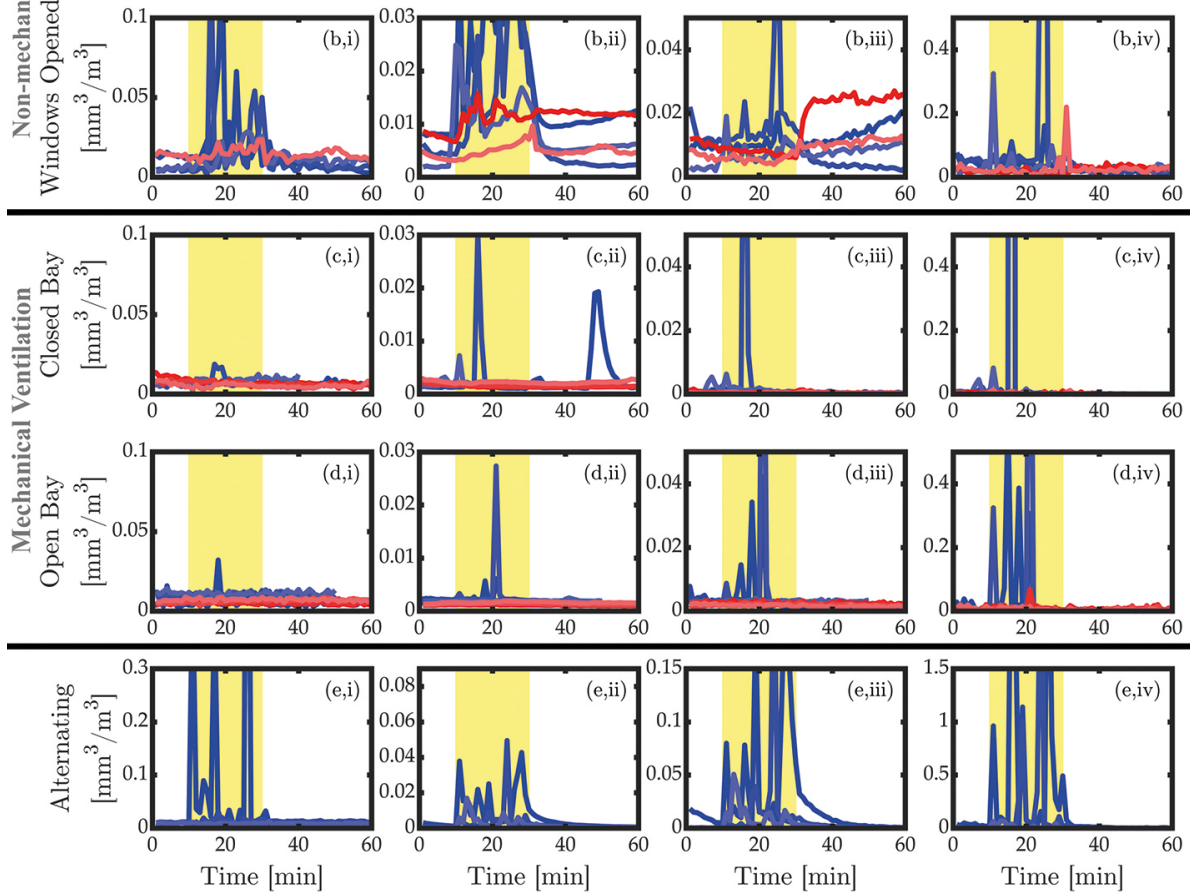

Fig. 2 Variation in measured aerosol levels for the same procedure (20 minutes drilling using FHP) repeated under different conditions. The blue lines represent recordings without EOS and red lines denote those including EOS. The columns represent the different size ranges of particles: i) 'very small' $(0.08-0.26 \mu \mathrm{m})$; ii) 'small' $(0.27-0.90 \mu \mathrm{m})$; iii) 'medium' (0.91-2.70 $\mu \mathrm{m})$; and iv) 'large' (2.71-10 $\mu \mathrm{m})$. The rows correspond to different surgery setup: a) nonmechanically ventilated environment with closed windows; b) non-mechanically ventilated environment with windows opened at end of the procedure; $c$ ) mechanically ventilated environment in a hospital closed surgery; d) open hospital clinic with mechanically ventilated environment, e) in which the tooth being drilled was alternated every five minutes. Yellow shaded regions indicate the duration of the procedure; the time preceding represents the initial pre-treatment period and the time after represents the post-treatment FT. (Note the limits of the $y$-axes in panel e are three times higher than those of other rows) produced by a fast handpiece (FHP), the minimum concentration of aerosols found for each size in the pre-procedural tenminute period was subtracted from the corresponding median value found during the procedure.

For the purpose of external validity, the experiment was repeated to measure size distribution of aerosol particles using a FHP for seven minutes on human extracted teeth and compared with plastic teeth under identical conditions in the non-mechanically ventilated environment with closed windows.

\section{Statistics}

Descriptive analysis was used to identify the characteristic in aerosol change in the various particle size groups; these were then represented visually in frequency graphs showing concentration $(\mathrm{mm} 3 / \mathrm{m} 3)$ over time (minutes) with the procedure period highlighted in yellow.

Further statistical analysis included the calculation of the post-procedure FT. This was represented by, the time when the particle concentration (in each particle size group) reached a threshold within a $5 \%$ of the mean of the pre-procedural particle con centration.

\section{Results}

\section{Aerosol generation and effect of} extraoral suction

Figure 2 summarises the aerosols measured throughout each of the experiments. These involved the use of a FHP for 20 minutes simulating the same procedure but under a variety of clinical conditions (yellow shaded region), both without (blue) and with (red) use of the EOS.

Aerosol levels were highest and most sustained in the non-mechanically ventilated environment with the windows closed throughout (Fig. 2a). There was a distinct increase in the concentration of aerosols across the 'very small', 'small' and 'medium' ranges during the procedure. In some cases, this increase occurred in the form of large, isolated spikes that arose apparently randomly (Fig. 2ai) at ten minutes and Figure 2aiii at 32 minutes. Apart from these spikes, there was a clear tendency for the concentration to gradually increase throughout the procedure and then slowly decline afterwards. This was most apparent in the 'small' size range (Fig. 2aii), where in some cases the concentration did not return 
Table 1 Estimated FT (in minutes) required for aerosol levels in each particle size range to return to within $5 \%$ of their initial concentration, for each experiment. The rightmost column shows the largest FT identified in each experiment. In some cases, the aerosol levels never returned to within $5 \%$ of their original level; these cases are denoted as ' $>X$ ', where $X$ is the time taken until the end of the measurement

\begin{tabular}{|c|c|c|c|c|c|c|}
\hline \multirow{2}{*}{ Procedure } & \multirow{2}{*}{ Number } & \multirow{2}{*}{$\begin{array}{l}\text { Very small } \\
(0.08- \\
0.26 \mu \mathrm{m})\end{array}$} & \multirow{2}{*}{$\begin{array}{l}\text { Small } \\
(0.27- \\
0.90 \mu \mathrm{m})\end{array}$} & \multirow{2}{*}{$\begin{array}{l}\text { Medium } \\
(0.91- \\
2.60 \mu \mathrm{m})\end{array}$} & \multirow{2}{*}{$\begin{array}{l}\text { Large } \\
(2.61- \\
10 \mu \mathrm{m})\end{array}$} & \multirow{2}{*}{$\begin{array}{l}\text { Overall } \\
\text { (minutes) }\end{array}$} \\
\hline & & & & & & \\
\hline \multirow{7}{*}{$\begin{array}{l}\text { Windows } \\
\text { closed }\end{array}$} & 1 & 17 & $>30$ & 1 & 1 & $>30$ \\
\hline & 2 & 1 & $>41$ & 1 & 2 & $>41$ \\
\hline & 3 & 8 & $>60$ & 8 & 16 & $>60$ \\
\hline & 4 & 13 & 27 & 10 & 2 & 27 \\
\hline & 5 & 36 & 31 & 12 & 1 & 36 \\
\hline & \multicolumn{5}{|l|}{ Median } & $>30$ \\
\hline & \multicolumn{5}{|l|}{ Max } & $>60$ \\
\hline \multirow{3}{*}{$\begin{array}{l}\text { Windows } \\
\text { closed } \\
\text { (with EOS) }\end{array}$} & 1 & 2 & 24 & 1 & 1 & 24 \\
\hline & \multicolumn{5}{|l|}{ Median } & 24 \\
\hline & \multicolumn{5}{|l|}{ Max } & 24 \\
\hline \multirow{5}{*}{ Closed bay } & 1 & 1 & 1 & 8 & 3 & 8 \\
\hline & 2 & 2 & 1 & 6 & 1 & 6 \\
\hline & 3 & 2 & 7 & 4 & 1 & 7 \\
\hline & \multicolumn{5}{|l|}{ Median } & 7 \\
\hline & \multicolumn{5}{|l|}{ Max } & 8 \\
\hline \multirow{5}{*}{$\begin{array}{l}\text { Closed bay } \\
\text { (with EOS) }\end{array}$} & 1 & 1 & 1 & 3 & 1 & 3 \\
\hline & 2 & 1 & 1 & 4 & 2 & 4 \\
\hline & 3 & 1 & 1 & 5 & 5 & 5 \\
\hline & \multicolumn{5}{|l|}{ Median } & 4 \\
\hline & \multicolumn{5}{|l|}{ Max } & 5 \\
\hline \multirow{7}{*}{ Open bay } & 1 & 1 & 1 & 2 & 7 & 7 \\
\hline & 2 & 17 & 3 & 2 & 2 & 17 \\
\hline & 3 & 1 & 1 & 1 & 1 & 1 \\
\hline & 4 & 5 & 1 & 1 & 1 & 5 \\
\hline & 5 & 2 & 1 & 2 & 1 & 2 \\
\hline & \multicolumn{5}{|l|}{ Median } & 5 \\
\hline & \multicolumn{5}{|l|}{ Max } & 17 \\
\hline \multirow{6}{*}{$\begin{array}{l}\text { Open bay } \\
\text { (with EOS) }\end{array}$} & 1 & 2 & 1 & 1 & 1 & 2 \\
\hline & 2 & 1 & 1 & 1 & 2 & 2 \\
\hline & 3 & 24 & 1 & 1 & 1 & 24 \\
\hline & 4 & 1 & 2 & 1 & 1 & 2 \\
\hline & \multicolumn{5}{|l|}{ Median } & 2 \\
\hline & \multicolumn{5}{|l|}{ Max } & 24 \\
\hline \multirow{5}{*}{$\begin{array}{l}\text { Alternating } \\
\text { handpiece } \\
\text { direction }\end{array}$} & 1 & 2 & 12 & 8 & 3 & 12 \\
\hline & 2 & 6 & $>30$ & 26 & 6 & $>30$ \\
\hline & 3 & 1 & 26 & 18 & 1 & 26 \\
\hline & Median & & & & & 26 \\
\hline & Max & & & & & $>30$ \\
\hline
\end{tabular}

to baseline levels up to 90 minutes after the procedure finished (Table 1).

When the EOS was used (red), the magnitude of the increase in aerosol particles during the procedure was reduced. The signals contain fewer large spikes and appeared to take less time to revert to baseline levels. In some cases (the 'medium' and 'large' size ranges), the use of the EOS resulted in a continuous reduction in aerosol levels throughout the entire experiment, indicating that the EOS was filtering out other background aerosols, as well as those produced by the operative procedure.

The equivalent data with the windows opened immediately following the end of the procedure (at 30 minutes) and left open during the whole post-procedure FT in the non-mechanically ventilated environment is illustrated in Figure 2b. During the procedure, the use of the EOS led to lower aerosol levels. In the case of the 'very small' and 'small' particles, the opening of the window coincided with a sudden reduction in aerosol levels, while in one case there was a sharp increase (Fig. 2biii).

When the procedures were repeated in the mechanically ventilated environment of the dental hospital, there was a distinctly different pattern. The closed bay (approximately six $\mathrm{ACH}$ ) exhibited lower pre-procedural baseline particle levels, with notably fewer spikes and no appreciable increase in concentration over the course of the procedure (Fig. 2c) compared with the non-mechanically ventilated environment in the practice setting. No clear differences were observed for the test performed in the open bay in the mechanically ventilated environment of the hospital (Fig. 2d).

The additive effect of the EOS was less noticeable in the hospital within a highefficiency particulate absorbing (HEPA)filtered mechanical ventilation environment during the open and closed bay experiments, implying that the ventilation system was sufficiently effective. With the exception of a minor small spike at 20 minutes in one instance (Fig. 2div), no evident large particle spikes were recorded during the AGP when the EOS was used, suggesting that EOSs could effectively prevent or reduce the frequency of high levels of particles generated by aerosol.

We hypothesised that these particle spikes occurred due to the relative proximity of the FHP to the intraoral suction device inlets 

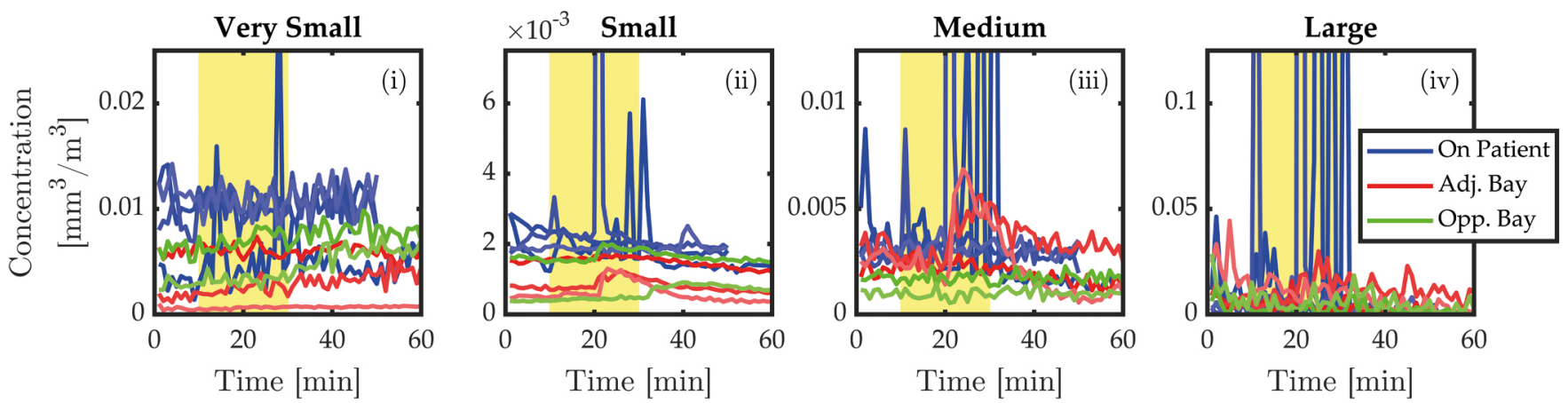

Fig. 3 Variation in measured aerosol levels for the same procedure (20 minutes drilling using FHP) performed in an open bay with additional measurements taken in the opposite and adjacent bays, measured at various positions (with EOS). The columns represent the different size

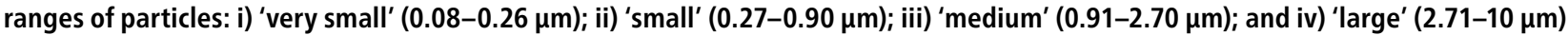

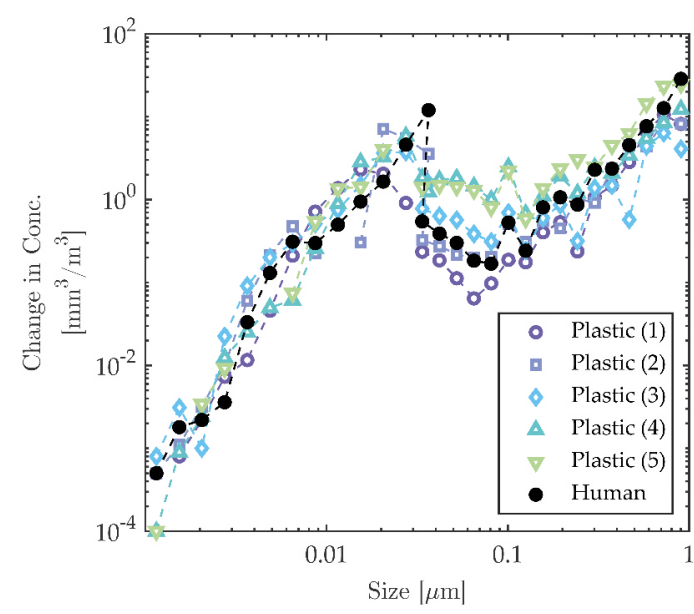

Fig. 4 Change in concentration of aerosol levels, recorded over seven minutes of drilling using FHP, relative to the minimum found in the ten minutes' pre-procedural time. The black symbols denote tests done using human teeth, while the other symbols correspond to repeated tests performed using plastic teeth (the results of these tests are also shown in Figure $2 \mathrm{a}$ )

(HVS and SE) and the water spray was not being effectively removed when moved or repositioned in the mouth. In order to verify this effect, we repeated the FHP procedure without the EOS, but changed the tooth that was being operated on every five minutes, alternating between the upper and lower anterior teeth (Fig. 2e). A very large increase in aerosol levels at five-minute intervals was observed, corresponding to the change in FHP position. It should be noted that the limits of the y-axes in Figure 2e are three times greater than the corresponding graphs discussed previously. This confirmed that brief changes in the position of the FHP, HVS or SE can lead to the release of large amounts of aerosol into the near vicinity.

\section{Open bay procedures}

In order to further investigate the behaviour of aerosols released during AGPs in open bay clinics, the procedure was repeated a number of times with the particle counters placed at adjacent and opposite bays. This effectively measured the potential for aerosol generated at one bay to lead to transmission to a patient or practitioner in a nearby bay. The time series for the various particle size ranges are shown in Figure 3 for measurements performed on the operative chair, at an adjacent and opposite bay.

A series of spikes in aerosol concentration were observed during the procedure near the patient's mouth. A minimal increase in medium particle size was observed in the adjacent bay. It is important to note the concentration is extremely low (y-axis) compared to the spike concentration on the patient.

\section{Tests with human teeth}

The size distribution of aerosol particles measured over seven minutes of operating on both human and plastic teeth under identical conditions (in a private practice with closed windows) was also assessed (Fig. 4). In order to identify the aerosols actually produced by the FHP, the minimum concentration of aerosols found for each size in the preprocedural ten-minute fallow period was subtracted from the corresponding median value found during the procedure. Little difference in the concentration with plastic and human extracted tooth tissue was observed (Fig. 4).

\section{Fallow time calculation}

A key question when considering how dentistry can safely be resumed during the pandemic is how much FT is required at the end of a given procedure. This was estimated from the aerosol measurements (Fig. 2) by calculating how long it took from the end of the procedure for the aerosol concentration in each size range to revert to within a threshold of $5 \%$ of the mean value before the procedure. A conservative approach was adopted, with the overall FT taken as the longest identified for each particle size range (Table 1). With the exception of two cases, the FT estimates differed by less than three minutes when threshold values of $0.05 \%$ and $7.5 \%$ were used (with 15 out of 23 cases showing no change), indicating that these estimates were not sensitive to the threshold value chosen.

This method was not applied to the experiments in which the windows were opened at the end of the procedure because, in these cases, the change in the aerosol levels measured were reflective of those outside the window, and in some cases, the air exchange led to a significant increase in the concentration (for example, Figure 2biii). Therefore, increases in aerosol concentration post-procedure in these experiments would not be associated with an increased risk of infection. 
The estimates of the overall FT contained significant scatter and some clear outliers in keeping with highly variable data (Fig. 2). The largest FT was found in the case of the non-mechanically ventilated environment with windows closed throughout, where in some cases, the concentration levels had not returned to baseline after more than an hour. The estimates for the required FT were notably smaller for the procedures in the hospital mechanically ventilated closed and open bays. With two exceptions, the aerosol levels were found to return to pre-procedure levels within less than ten minutes. This demonstrated the effectiveness of the ventilation system at reducing the required FTs, at least when operated at six $\mathrm{ACH}$.

FT estimates were larger for the procedures in which the tooth being operated on was alternated every five minutes (Fig. 2), indicating that even with mechanical ventilation, the FT required was dependent on the procedure being carried out.

\section{Discussion}

There is no published research evaluating aerosol procedures within dentistry which directly relates to contemporary practice in both hospital and private practice settings. This is of particular importance with the current return to practice initiatives across the world and the development of guidelines for dentists. Previously published studies represent the behaviour of droplets (splatter) in dental clinics after AGPs, rather than considering aerosol (of $<5 \mu \mathrm{m}){ }^{2}$ Moreover, a recent literature review concluded that the current evidence base cannot support a defined and appropriate FT for dental AGPs in the context of the COVID19 pandemic with very weak evidence that peaks in bacterial dissemination during dental procedures, which may take approximately 30 minutes to dissipate. ${ }^{24,26}$

Our findings indicate that a key arbiter of the delivery of safe dental care during COVID-19 is ventilation. We noted a marked decrease in aerosol in the locality with the use of HEPA-filtered mechanical ventilation, opening of windows and the use of an EOS. The impact of ventilation was best represented during the procedures in practice with nonmechanical ventilation when windows were left closed throughout, with an increase in the concentration of aerosols across all size ranges during the procedure followed by a slow decline during the FT. In some instances, the FT exceeded 90 minutes and therefore our results: 1) strongly indicate that AGPs should not be carried out in surgeries without ventilation; and 2) corroborate the findings of a recently published scoping review. ${ }^{24}$ Figure 2aiii demonstrates the risk of undertaking AGPs in a non-ventilated surgery. The procedures were carried out back to back without any air change between procedures, and with each subsequent experiment, the level of concentration increased. In practice, this would translate to an increasing risk of transmission to patient and staff after every AGP.

When windows were left open after the procedure, effective exchange between the indoor and outdoor air was observed, and the increase in concentration would not represent an increased risk of transmission, as the outside air is most likely a representative of outdoor pollutants. ${ }^{27}$ This suggests effective dilution of aerosol by natural ventilation after opening a window at the end of an AGP. Natural ventilation, such as a window, allows outside air to mix with room air to dilute any aerosol; however, it is claimed that it is not possible to quantify the number of $\mathrm{ACH}$ due to variation in effectiveness of dilution and so this would impact on calculation of FT. ${ }^{24}$ Theoretical modelling of airborne contaminants has been reported to predict $\mathrm{FT}$ at a wide range of air change rates, for AGPs of varying lengths, and with or without procedural mitigation. ${ }^{24}$ The modelling makes a number of assumptions, ${ }^{28}$ including that all procedures would generate aerosolised virus at the same rate, and that aerosols and larger droplets produced by dental procedures will only be removed by dilution. The accuracy and validity of such tools is difficult to ascertain as the algorithm and data used for calculation is unclear and not publicly available.

In this study, mechanical ventilation with six $\mathrm{ACH}$, such as those in most modern hospital environments, showed low preprocedural particulate counts at all particle sizes. Modern systems appear to require lower FT due to their efficiency, and more importantly, reduced risk to operators and those in the nearby surroundings. This study corroborated the findings of a previous study in which splatter from AGPs did not show any difference in distribution between open clinic and closed surgery environments. ${ }^{2}$ Although there were small spikes recorded in the adjacent bay, the increases tended to be broad (that is, not dominated by isolated spikes) and occurred 10-20 minutes after the start of the procedure. These were not repeatable and seemed random, so would be considered less relevant. These effects were likely to be a result of gradual dispersion of aerosol from the patient. Diffusion homogenises the concentration of particles, leading to a reduction in the magnitude of spikes, which was confirmed in that the emissions from the procedures themselves far exceed those from any other non-dental source.

Mitigation has been reported in national and international guidelines and documents produced by working groups as a method of reducing aerosol. Most guidelines are, however, largely based on outdated research or data pertaining to splatter rather than true aerosol. ${ }^{2}$ The National Services Scotland technical report indicated that ten minutes was necessary to allow droplets ( $>5-10 \mu \mathrm{m})$ to settle, regardless of air change rate, and that standard infection control precautions, which are well rehearsed in dental practice, are sufficient to mitigate the hazard. ${ }^{22}$ Previously, the use of EOSs has demonstrated reduction of splatter (intensity of contamination and frequency) during most of the simulated procedures in both open clinic bays and closed bays, in the same mechanically ventilated setting as used in this study. ${ }^{2,21}$ In another recent study, an EOS was found to statistically significantly reduce the aerosol particulate levels during various AGPs. ${ }^{29}$ Another contemporary study shows that modern aerosol management systems are effective at reducing aerosol sufficiently so as to require no FT; however, they are still not sufficient to reduce intra-operative aerosol generation, so the authors still suggest the use of appropriate PPE. ${ }^{30}$

In this study, the additive effect of EOSs on aerosol in reducing the FT in a nonmechanically ventilated environment was confirmed. This was unsurprising given the lack of any mechanical ventilation other than the EOS. However, the additive effect of the EOS was less noticeable in the hospital environment involving HEPA-filtered mechanical ventilation, implying that the ventilation system in isolation was potent. The EOS was less likely to reduce the median overall FT in an open bay environment. This reflects the fact that in a closed bay, the effect of the EOS on the overall ACH was larger than in the open bay characterised by a larger area and a greater number of ventilation ports. Notwithstanding this, the particle spikes recorded during the AGP were less noticeable in both open and closed bays when using an 
EOS, and without an EOS, these small spikes represented the majority of the increase in aerosol levels.

The data from this study is accurate for the air filtration system within our dental hospital (six $\mathrm{ACH})$ and variation between filtration systems of different makes, models and age may be more or less effective than the system tested.

Given the rapid evaporation of very small droplets, the aerosols can be expected to comprise primarily solid droplet nuclei such as tiny fragments of the tooth. ${ }^{31}$ The production of these fragments is likely to depend on the physical properties of the tooth, any restorative material and the cutting action at the chipping interface. Undertaking simulation on extracted natural teeth under the same conditions as the plastic manikin teeth identified a lack of variation in particle characteristics, and therefore, the results of this study can be interpreted with confidence as being representative of a real-world scenario.

Dentists have access to a large variety of dental equipment, and there will undeniably be variation in the aerosol production between these products and their mode of use. For example, not only would we expect differences in the amount of water coolant released during FHP use, but also the force at which this is released and the subsequent spread of this into the environment. FT estimates were larger for the procedures in which the tooth being drilled was alternated every five minutes. This suggested that the position and strength of suction is vital in reducing aerosol levels, and optimal techniques are recommended whereby suction is placed at the operating site before the operation of the FHP, maintaining an intimate relationship when moving between operating sites. Equally, it would be sensible to stop the handpiece before changing position to reduce the escape of aerosol from the suction inlets.

Furthermore, as with all experiments of this nature, all results are specific to the operators and assistants conducting the procedures. As such, there will undoubtedly be variations in aerosol production associated with different operators and assistants. The procedures were conducted using a manikin, thereby eliminating patient factors such as movement, saliva, tongue and involuntary actions, which may have an impact on the amount of saliva generated in real patients. The authors suggest that future research specifically looks at the aerosolisation of respiratory viruses and the associated FTs.

\section{Conclusions}

Within the limitations of this study, the following conclusions can be drawn:

1. Ventilation in dental practices is an essential prerequisite for carrying out AGPs; no AGP should be carried out in rooms without ventilation

2. Recommended FT in a HEPA-filtered mechanically ventilated room with at least six ACH may be as little as ten minutes

3. The EOS system reduced the peaks in particle concentration in non-mechanically ventilated and mechanically ventilated environments

4. Careful four-handed dentistry with HVS and SE appears to remain the primary mitigating method.

\section{Conflict of interest}

There are no known conflicts of interest. Testing equipment was provided by TSI Incorporated and their involvement with this study was on an advisory basis only and had no bearing on the outcomes achieved.

\section{Acknowledgements}

TSI Incorporated, USA. Specialist Dental Services, Harley Street, UK. For allowing us access to a primary care setting for non-mechanically ventilated surgeries representative of most dental provision in the UK.

\section{Author contributions}

All authors gave their final approval and agree to be accountable for all aspects of the work. Shakeel Shahdad: contributed to conception, design, data acquisition and interpretation, drafted and critically revised the manuscript. Annika Hindocha: design, data acquisition, drafted and critically revised the manuscript. Tulsi Patel: design, data acquisition, drafted and critically revised the manuscript. Neil Cagney: contributed to conception, design, interpretation, drafted and critically revised the manuscript. Jens-Dominik Mueller: contributed to conception, design, interpretation and critically revised the manuscript. Amine Koched: contributed to conception and interpretation. Noha Seoudi: contributed to conception, design, interpretation and critically revised the manuscript. Claire Morgan: contributed to conception and critically revised the manuscript. Padhraig Fleming: contributed to conception, design, interpretation and critically revised the manuscript. Ahmed Riaz Din: contributed to conception, design, data acquisition and interpretation, drafted and critically revised the manuscript.

\section{References}

1. Allison J R, Currie C C, Edwards D C et al. Evaluating aerosol and splatter following dental procedures: Addressing new challenges for oral health care and rehabilitation. J Oral Rehabil 2021; 48: 61-72.

2. Shahdad S, Patel T, Hindocha A et al. The efficacy of an extraoral scavenging device on reduction of splatter contamination during dental aerosol generating procedures: an exploratory study. Br Dent J 2020; DOI: 10.1038/s41415-020-2112-7.

3. World Health Organisation. Transmission of SARSCoV-2: implications for infection prevention precautions 2020. Available at https://www.who.int/news-room/ commentaries/detail/transmission-of-sars-cov-2implications-for-infection-prevention-precautions (accessed April 2021).

4. Li Y, Qian H, Hang J et al. Evidence for probable aerosol transmission of SARS-CoV-2 in a poorly ventilated restaurant. 2020. Available online at https://www. medrxiv.org/content/10.1101/2020.04.16.20067728v1 (accessed April 2021).

5. Miller S L, Nazaroff W W, Jimenez J L et al. Transmission of SARS-CoV-2 by inhalation of respiratory aerosol in the Skagit Valley Chorale superspreading event. Indoor Air 2021; 31: 314-323.

6. Leggat P A, Kedjarune U. Bacterial aerosols in the dental clinic: A review. Int Dent J 2001; 51: 39-44.

7. Harrel S K, Molinari J. Aerosols and splatter in dentistry: A brief review of the literature and infection control implications. J Am Dent Assoc 2004; 135: 429-437.

8. Zemouri C, de Soet H, Crielaard W, Laheij A. A scoping review on bio-aerosols in healthcare and the dental environment. PLoS One 2017; DOI: 10.1371/journal. pone.0178007.

9. Bentley CD, Burkhart N W, Crawford J J. Evaluating spatter and aerosol contamination during dental procedures. J Am Dent Assoc 1994: 125: 579-584.

10. Teanpaisan $R$, Taeporamaysamai M, Rattanachone $P$, Poldoung N, Srisintorn S. The usefulness of the modified extra-oral vacuum aspirator (EOVA) from household vacuum cleaner in reducing bacteria in dental aerosols. Int Dent J 2001; 51: 413-416.

11. Chuang $C Y$, Cheng $H C$, Yang S, Fang W, Hung

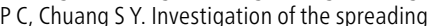
characteristics of bacterial aerosol contamination during dental scaling treatment. J Dent Sci 2014; 9 : 294-296.

12. Holloman J L, Mauriello S M, Pimenta L, Arnold R R. Comparison of suction device with saliva ejector for aerosol and spatter reduction during ultrasonic scaling. J Am Dent Assoc 2015; 146: 27-33.

13. Al-Amad S H, Awad M A, Edher F M, Shahramian K, Omran T A. The effect of rubber dam on atmospheric bacterial aerosols during restorative dentistry. J Infect Public Health 2017; 10: 195-200.

14. Zemouri C, Volgenant C M C, Buijs M J et al. Dental aerosols: microbial composition and spatial distribution. J Oral Microbiol 2020; DOI: 10.1080/20002297.2020.1762040

15. Mirhoseini S H, Koolivand A, Bayani M et al. Quantitative and qualitative assessment of microbial aerosols in different indoor environments of a dental school clinic. Aerobiologia (Bologna) 2021; DOI: 10.1007/ s10453-020-09679-z.

16. Chiramana S, Hima Bindu S O, Kishore Kadiyala K, Prakash M, Durga Prasad T, Krishna Chaitanya S. Evaluation of Minimum Required Safe Distance between Two Consecutive Dental Chairs for Optimal Asepsis. J Orofac Res 2013; 3: 12-15.

17. Veena H R, Mahantesha S, Joseph PA, Patil SR, Patil $\mathrm{SH}$. Dissemination of aerosol and splatter during ultrasonic scaling: A pilot study. J Infect Public Health 2015; 8: 260-265

18. Junevicius J, Surna A, Surna R. Effectiveness evaluation of different suction systems. Stomatologija 2005; 7 : 52-57.

19. Polednik B. Aerosol and bioaerosol particles in a dental office. Environ Res 2014: 134: 405-409.

20. Bradna P, Ondrackova L, Zdimal V, Navratil T, Pelclova D. Detection of nanoparticles released at finishing of dental composite materials. Monatsh Chem 2017; 148: 531-537. 
21. Din A R, Hindocha A, Patel T et al. Quantitative analysis of particulate matter release during orthodontic procedures: a pilot study. Br Dent J 2020; DOI: 10.1038/ s41415-020-2280-5.

22. National Services Scotland Short Life Working Group. SBAR Ventilation, water and environmental cleaning in dental surgeries relating to COVID-19. 2020. Available at https://www.scottishdental.org/wp-content/ uploads/2020/08/Ventillation-Final-Copy-1.pdf (accessed April 2021).

23. Faculty of General Dental Practice (UK). Implications of COVID-19 for the safe management of general dental practice: A practical guide. 2020. Available at https://www.fgdp.org.uk/sites/fgdp.org.uk/files/ editors/FGDP\%20CGDent\%20Implications\%20of\%20 COVID-19\%20for\%20the $\% 20$ safe $\% 20$ management $\% 20$ of $\% 20$ general $\% 20$ dental $\% 20$ practice $\% 202 \% 20$ October\%202020\%20v2.pdf (accessed April 2021).
24. Scottish Dental Clinical Effectiveness Programme. Mitigation of Aerosol Generating Procedures in Dentistry: A Rapid Review. 2021. Available at https:// www.sdcep.org.uk/wp-content/uploads/2021/04/ SDCEP-Mitigation-of-AGPs-in-Dentistry-Rapid-Reviewv1.1.pdf (accessed April 2021).

25. Clarkson J, Ramsey C, Richards D, Robertson C, AceveMartins M. Aerosol Generating Procedures and their Mitigation in International Dental Guidance Documents -A Rapid Review. 2020. Available at https://oralhealth. cochrane.org/sites/oralhealth.cochrane.org/files/public/ uploads/rapid_review_of_agps_in_international_ dental_guidance_documents.pdf (accessed April 2021).

26. Innes N, Johnson I G, Al-Yaseen W et al. A systematic review of droplet and aerosol generation in dentistry. J Dent 2021; 105: 103556.

27. Gouriou F, Morin J P, Weill M E. On-road measurements of particle number concentrations and size distributions in urban and tunnel environments. Atmos Environ 2004 38: $2831-2840$

28. Faculty of General Dental Practice (UK). Dental Fallow Time Calculator. 2020. Available at https://www.fgdp. org.uk/news/dental-fallow-time-calculator-launched (accessed April 2021).

29. Nulty A, Lefkaditis C, Zachrisson P, van Tonder Q, Yar R. A clinical study measuring dental aerosols with and without a high-volume extraction device. Br Dent J 2020; DOI: 10.1038/s41415-020-2274-3.

30. Ehtezazi T, Evans D G, Jenkinson I D et al. SARS-CoV-2: characterisation and mitigation of risks associated with aerosol generating procedures in dental practices. $\mathrm{Br}$ Dent J 2020; DOI: 10.1038/s41415-020-2274-3.

31. Xie X, Li Y, Chwang A T Y, Ho P L, Seto W H. How far droplets can move in indoor environments - revisiting the Wells evapouration-falling curve. Indoor Air 2007; 17: $211-225$. 